rate in healthy young individuals in the recumbent position, the effects are very marked in erect position, and consist, after an initial rise in systolic blood pressure, in a considerable drop which may lead to syncope. The pulse rate may show a similar change or may remain increased during the whole experiment. In the presence of carbon dioxide, however, the drop in blood pressure is prevented and may be maintained normal or may even be elevated. Obviously, carbon dioxide, by increasing respiration and by its effect on the circulation, provides a better blood supply to the brain, which is apparent in the erect position, because under these conditions the effects of oxygen lack become more severe, since a temporary cerebral anæmia frequently results from maintaining an erect position under oxygen lack.

Department of Physiology, ERNST GelLhorn. College of Medicine,

University of Illinois, Chicago. Feb. 22.

${ }^{2} \mathrm{~S}$. B. Childs, H. Hamlin and $\mathrm{Y}$. Henderson, NATURE, 135, 457 (1935) "A. Mosso, "Life of Man on the High Alpes"' (London, 1898).

${ }^{3}$. Margaria, Areh. Sei. biol., 11, 425 (1928).

'C. Talenti, Arch. Sci. biol., 14, 125 (1930).

Margaria, Arch. Sci. biol., 11, 453 (1928).

' E. Gellhorn and I. Spiesman, Amer. J. Physiol., 112, 519, 620 and $662(1935)$.

${ }^{7}$ E. Gellhorn and S. Kraines, Science, 1936, in the press.

\section{Plasticity of Bismuth Crystals}

MUCH evidence of a conflicting nature has been advanced on the subject of the plasticity of bismuth : the following brief account of some new experiments may therefore be of interest. torsional stresses ${ }^{1}$, it was found that the crystals deformed entirely by twinning without any slip. The non-occurrence of slip in these tests appeared to be at variance with the conclusions drawn by other workers as to the behaviour of bismuth ${ }^{2}$; but it appeared possible that the difference might be due to the special nature of the applied stresses. Accordingly a third single crystal of bismuth was tested under cycles of reversed direct stresses (tension and compression). This specimen also deformed only by twinning; the final fracture was by cleavage parallel to the plane 111 (0001 in hexagonal co-ordinates), but the only visible signs of movement that could be termed slip were a few faint bands parallel to this plane (111) in the neighbourhood of the fracture. The ratio of the shear stress to the normal stress on the plane 111 of this crystal was approximately unity, so that the rule suggested by Georgieff and Schmid-that slip should occur before cleavage if this ratio is greater than $0 \cdot 7$-was not confirmed. Still more recently, a portion of this third crystal has been tested under static compression force applied parallel to the same axis as in the alternating direct stress tests. In this test the crystal deformed almost entirely by slip parallel to the plane 111, no regular system of twin bands being produced.

The appearance of the slip bands produced by the slip deformation is shown in the accompanying photographs (Fig. 1) It may be noted that these slip bands are not very like the bands produced by slip in aluminium, silver, iron, etc., and that in fact they appear much more like twin bands; comparison may be made with the twin bands (on the planes of the type (011)), which are also shown in the bottom right-hand photograph. Although it was not practicable in the compression test to deform the specimen sufficiently definitely to establish the shear nature of the deformation, there is very little doubt that the de. formation as a whole was pure shear parallel to the plane 111. Berg ${ }^{3}$ has suggested that the occurrence of slip in single crystals of bismuth may depend to some extent upon the applied stress conditions and upon the manner in which the single crystals are grown. The results of our tests confirm that slip may be produced in bismuth under certain types of stress system; but it appears that deformation by twinning may often occur more easily.

\section{H. J. GOUgh.}

H. L. Cox.

National Physical

Laboratory,

Teddington, Middlesex.
In an investigation carried out at the National Physical Laboratory on the behaviour of two single crystals of bismuth when subjected to alternating
'H. J. Gough and H. L. Cox, J. Inst. Met., 48, No. 1 (1932). 2 M. Georgleff and E. Schmid, $Z$. Phys., 36, 759 (1926), 